\title{
Estimativa das temperaturas cardinais e modelagem do desenvolvimento vegetativo em batata-doce
}

\author{
Lígia Erpen', Nereu A. Streck', Lilian O. Uhlmann'1, \\ Josana A. Langner' ${ }^{1}$, José E. M. Winck ${ }^{1}$ \& Luana F. Gabriel ${ }^{1}$
}

\begin{abstract}
RESUMO
Objetivou-se, neste trabalho, determinar as temperaturas cardinais da batata-doce e comparar a simulação da emissão de nós com o modelo do plastocrono (linear) e com o modelo de Wang e Engel (não linear) além da melhor forma de entrada da temperatura do ar nos modelos. A calibração e o teste dos modelos foram feitos pelos dados de número de nós na haste principal de plantas de batata-doce, cultivar Princesa, coletados em experimentos conduzidos em Santa Maria, RS, Brasil, em sete épocas de plantio, nos anos 2010, 2011 e 2012. Melhor predição dos modelos foi obtida com o uso das temperaturas cardinais 12, 30 e $40{ }^{\circ} \mathrm{C}$. Os modelos plastocrono e Wang e Engel apresentaram desempenho semelhante. A versão com a temperatura média foi superior às temperaturas mínima e máxima, em ambos os modelos. Os dois modelos podem ser utilizados para simular o desenvolvimento vegetativo da batata-doce quando cultivada na época recomendada. Sugere-se, fora deste período, o uso do modelo de Wang e Engel.
\end{abstract}

Palavras-chave: Ipomoea batatas, fenologia, plastocrono, temperatura do ar

\section{Estimating cardinal temperatures and modeling the vegetative development of sweet potato}

\begin{abstract}
The objective of this study was to determine the cardinal temperatures of sweet potato and to compare the simulation of node appearance with the plastochron model (linear) and with Wang and Engel model (nonlinear) and the best way to input air temperature in the models. Model calibration and test were conducted with data of number of nodes on the main stem of sweet potato, cultivar Princesa, collected in experiments carried out in Santa Maria, RS, Brazil, at seven planting dates in 2010, 2011 and 2012 growing seasons. Better model predictions were obtained using the cardinal temperatures of 12,30 and $40{ }^{\circ} \mathrm{C}$. The plastochron and Wang and Engel models showed similar performance. The version of the models that uses mean temperature as input was superior to the version with the minimum and maximum temperatures as input. Both models can be used to simulate the development of sweet potatoes when it is cultivated in the recommended period. Outside this period it is suggested to use the Wang e Engel model.
\end{abstract}

Key words: Ipomoea batatas, phenology, plastochron, air temperature 


\section{INTRODUÇÃO}

A batata-doce (Ipomoea batatas (L.) Lam.) é uma planta nativa da América Central constituindo, atualmente, uma das principais culturas tuberosas produzidas em regiões tropicais e subtropicais de todo o mundo.O baixo custo de produção, a rusticidade do cultivo, o alto potencial produtivo e o valor alimentício da batata-doce, são características importantes desta cultura olerícola. Sua utilização é destinada sobretudo para a alimentação humana e animal, e como matéria-prima na indústria de alimento e para a produção de etanol (Castro, 2010).

Modelos de simulação das culturas permitem descrever as interações entre a planta e o ambiente. São ferramentas atualmente usadas para diferentes finalidades, como na determinação da época mais apropriada da aplicação de práticas de manejo e para estudar a resposta e a adaptação da agricultura às alterações do clima uma vez que podem quantificar as mudanças no ciclo das culturas em diferentes cenários climáticos (Streck et al., 2006; Walter et al., 2010).

O desenvolvimento vegetativo, representado pela taxa de aparecimento de nós (TAN), é uma parte significativa de modelos de simulação de culturas agrícolas (Streck et al., 2006). A integração da TAN no tempo fornece o número de nós (NN) acumulados, que está relacionado com diferentes fases de desenvolvimento da planta e com a evolução da área foliar, responsável pela interceptação da radiação solar e fotossíntese (Streck et al., 2003; Xue et al., 2004).

A temperatura é o principal fator que determina a TAN nas plantas, que é máxima sob temperatura ótima (Tot) e cessa quando a temperatura está abaixo ou acima da temperatura basal inferior ( $\mathrm{Tb}$ ) e basal superior (TB). Esses pontos na resposta do desenvolvimento à temperatura caracterizam as temperaturas cardinais de cada espécie (Yan \& Hunt, 1999). Para a batatadoce, diferentes valores são citados na literatura, principalmente para a $\mathrm{Tb}$. O desenvolvimento é máximo em temperaturas próximas a $30{ }^{\circ} \mathrm{C}$ (Silva \& Lopes, 1995 ; Villavicencio et al., 2007) e é paralisado em temperaturas acima de $40^{\circ} \mathrm{C}$ (Ravi et al., 2009) e em temperaturas baixas, com valores variando de 10 a $15^{\circ} \mathrm{C}$ (Silva \& Lopes, 1995; Noh et al., 2009; Villordon et al., 2009). Esta variação nos valores de temperaturas cardinais citados na literatura é uma dificuldade para a modelagem desta cultura havendo necessidade, portanto, de se determinar, com mais precisão, tais temperaturas.

A TAN pode ser estimada pelo plastocrono, definido como a soma térmica necessária para o aparecimento de dois nós sucessivos em uma haste (Baker \& Reddy, 2001). Embora seja um modelo muito utilizado por sua simplicidade, a soma térmica apresenta desvantagens, entre elas a pressuposição de que a resposta da TAN é linear à temperatura (Xue et al., 2004), o que ocorre apenas em uma faixa entre a Tb e a Tot.Em temperaturas próximas à $\mathrm{Tb}$ e acima da Tot, a resposta é melhor descrita de forma não linear (Streck et al., 2011). Assim, o plastocrono pode não ser o melhor modelo em situações em que a temperatura esteja fora do intervalo linear, como em datas de plantios precoces e tardias, quando temperaturas frias são frequentes e sob cenários de mudança climática, quando temperaturas mais altas também são frequentes (Streck et al., 2009).
Uma alternativa à soma térmica são os modelos multiplicativos e que empreguem funções de resposta não lineares, como o modelo de Wang e Engel (Wang \& Engel, 1998). A função de temperatura $\mathrm{f}(\mathrm{T})$ usada neste modelo contempla a faixa linear do desenvolvimento podendo, então, funcionar melhor nas diferentes situações e regiões de cultivo (Streck, 2003). Estudos indicam que este modelo tem melhor desempenho do que o método da soma térmica para descrever a emissão de folhas em outras culturas agrícolas, como kiwi (Streck, 2003), trigo (Xue et al., 2004), batata (Streck et al., 2007) e milho (Streck et al., 2008).

A temperatura é a principal variável ambiental de entrada nos modelos da soma térmica e na $\mathrm{f}(\mathrm{T})$. Há duas maneiras de entrada da temperatura: com a média entre a temperatura mínima e máxima diária do ar ou com cada valor de temperatura mínima e máxima diária (Tmm) (Xue et al., 2004). Essas duas versões podem render resultados distintos no desempenho dos modelos para diferentes culturas agrícolas; por exemplo, em milho houve melhora na predição da velocidade de aparecimento de folhas com a versão Tmm (Streck et al., 2009), mas em trigo a melhor predição foi obtida com o uso da Tmed (Xue et al., 2004). Portanto, as duas versões precisam ser testadas nos modelos de simulação do desenvolvimento na cultura da batata-doce.

O objetivo deste trabalho foi determinar as temperaturas cardinais da batata-doce e comparar a simulação da emissão de nós com o modelo do plastocrono (linear) e com o modelo de Wang e Engel (não linear) e a melhor forma de entrada da temperatura do ar nos modelos.

\section{Material e Métodos}

Experimentos de campo com a cultura da batata-doce foram conduzidos no Departamento de Fitotecnia da Universidade Federal de Santa Maria (Santa Maria, RS, latitude: $29^{\circ} 43^{\prime} \mathrm{S}$, longitude: $53^{\circ} 43^{\prime} \mathrm{W}$ e altitude: $95 \mathrm{~m}$ ) durante os anos de 2010, 2011 e 2012, em diferentes épocas de plantio, a fim de submeter as plantas a diversas condições térmicas durante a emissão de nós. O solo do local é uma transição entre a Unidade de Mapeamento São Pedro (Argissolo Vermelho Distrófico arênico) e a Unidade de Mapeamento Santa Maria (Alissolo Hipocrômico Argilúvico típico) (Streck et al., 2008a). Segundo a classificação de Köppen, o clima é do tipo Cfa, subtropical úmido com verões quentes e sem estação seca definida.

A cultivar de batata-doce utilizada foi a Princesa, da Embrapa Hortaliças. As mudas utilizadas no plantio foram produzidas em bandejas de 128 células com substrato comercial pelo enraizamento de hastes de batata-doce com três a quatro nós. O preparo do solo foi o convencional, com aração e gradagem e construção de camalhões de $30 \mathrm{a} 40 \mathrm{~cm}$ de altura. A adubação foi aplicada no camalhão antes do plantio, de acordo com a análise química do solo e recomendação técnica para a cultura. O plantio foi realizado quando as mudas tinham 6 a 8 folhas, no espaçamento de $1,0 \mathrm{~m}$ (entre camalhões) $\mathrm{x}$ $0,4 \mathrm{~m}$ (entre plantas). Na primeira semana após o transplantio foram realizadas irrigações diárias para facilitar o pegamento das mudas. Após este período foram realizadas irrigações suplementares quando havia longos períodos sem precipitação (10 a 15 dias) (Castro, 2010), de modo que não houvesse 
estresse hídrico. O controle de plantas daninhas foi manual, sempre que necessário.

O delineamento experimental foi blocos ao acaso com quatro repetições. Cada parcela foi constituída de três fileiras de $4 \mathrm{~m}$ de comprimento com 10 plantas por fileira, sendo as duas fileiras laterais como bordadura. As datas de plantio foram: 27/03/2010 (época 1), 18/10/2010 (época 2), 21/12/2010 (época 3), 25/08/2011 (época 4), 21/11/2011 (época 5), 03/01/2012 (época 6) e 13/02/2012 (época 7). Seis plantas foram marcadas na fileira central de cada parcela nas quais, uma ou duas vezes por semana, foi contado o número de nós visíveis na haste principal (NN). Um nó foi considerado visível quando a folha associada a ele apresentava as bordas do limbo foliar desenroladas e separadas. As avaliações foram realizadas até os dias 07/06/2010 (época 1), 27/02/2011 (época 2), 09/03/2011 (época 3), 14/12/2011 (época 4), 23/02/2012 (época 5), 02/04/2012 (época 6) e 14/05/2012 (época 7).

Os dados diários de temperatura mínima (TN) e máxima (TX) do ar e radiação solar global incidente foram coletados na Estação Climatológica Principal do $8^{\circ}$ Distrito de Meteorologia do Instituto Nacional de Meteorologia (Disme/Inmet), localizada a aproximadamente $100 \mathrm{~m}$ da área experimental.

Dois modelos foram utilizados para simular a emissão de nós em batata-doce: o modelo do plastocrono (PLASTO) e o modelo de Wang e Wengel (WE). A representação gráfica dos dois modelos está na Figura 1.

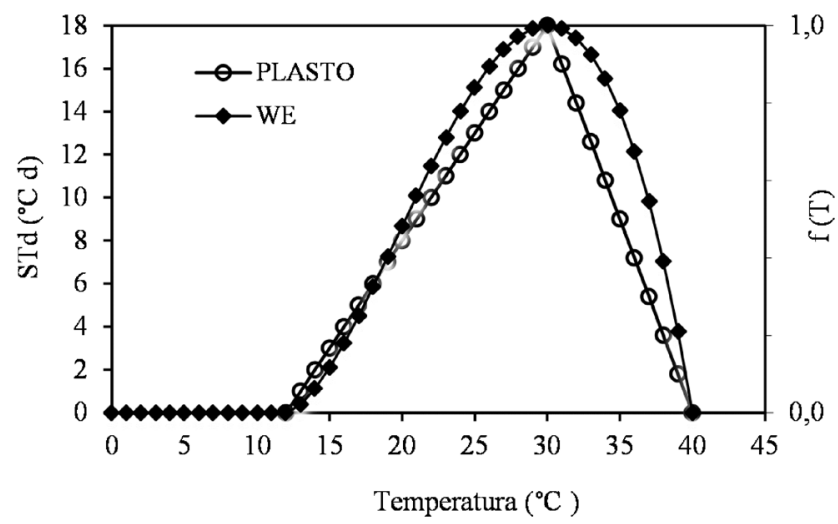

Obs.: STd é a soma térmica diária ( ${ }^{\circ} \mathrm{C}$ d) e f(T) é a função de resposta à temperatura

Figura 1. Representação gráfica da resposta da emissão de nós a temperatura do ar no modelo do plastocrono (PLASTO) e de Wang e Wengel (WE) com as temperaturas cardinais de 12,30 e $40{ }^{\circ} \mathrm{C}$

No modelo PLASTO o número de nós na haste principal (NN) foi calculado por:

$$
\mathrm{NN}=\frac{\mathrm{STa}}{\mathrm{PLAST}}\left(\text { nós haste }^{-1}\right)
$$

em que: STA é a soma térmica acumulada $\left({ }^{\circ} \mathrm{C}\right.$ d) e PLAST é o valor do plastocrono $\left({ }^{\circ} \mathrm{C} \mathrm{d}\right.$ nó $\left.{ }^{-1}\right)$ calculado pelo inverso do coeficiente angular da regressão linear entre o NN e STa, a partir da data em que as plantas foram marcadas (Baker \& Reddy, 2001). A STa foi calculada por Arnold (1960):

$$
\mathrm{STa}=\sum \operatorname{STd}\left({ }^{\circ} \mathrm{C} \mathrm{d}\right)
$$

em que:

$\Sigma \mathrm{STd}$ - Somatório das somas térmicas diárias (STd)

A soma térmica diária (STd) foi calculada por Arnold (1960):

$$
\mathrm{STd}=(\mathrm{T}-\mathrm{Tb})
$$

quando a $\mathrm{Tb}<\mathrm{T} \leq \mathrm{Tot}$

$$
\mathrm{STd}=\frac{(\mathrm{Tot}-\mathrm{Tb}) \cdot(\mathrm{TB}-\mathrm{T})}{\mathrm{TB}-\mathrm{Tot}}
$$

quando Tot $<\mathrm{T} \leq \mathrm{TB}$

em que: Tn, Tot e TB são as temperaturas cardinais para aparecimento de nós em batata-doce $\mathrm{e} \mathrm{T}$ é a temperatura diária do ar $\left({ }^{\circ} \mathrm{C}\right)$.

No modelo de WE o NN foi calculado por Wang \& Engel (1998) e Streck (2003):

$$
\mathrm{NN}=\sum \mathrm{TAN}\left(\text { nós } \text { haste }^{-1}\right)
$$

em que:

$\Sigma$ TAN - Somatório da taxa de aparecimento de nós diária

A taxa de aparecimento de nós diária (TAN) foi calculada por:

$$
\text { TAN }=\text { TANmax } . \mathrm{f}(\mathrm{T})
$$

em que: TANmax é a taxa diária máxima de aparecimento de nós $\left(\right.$ nós $\left.\mathrm{d}^{-1}\right)$ sob ótima temperatura e $\mathrm{f}(\mathrm{T})$ a função resposta à temperatura. A TANmax foi estimada pelo método dos mínimos quadrados, que minimiza os resíduos entre valores observados e simulados de NN (Xue et al., 2004). A função de temperatura $\mathrm{f}(\mathrm{T})$ no modelo de WE é uma versão da função beta, que varia de zero a um e definida como:

$$
f(T)=\frac{\left[2(T-T b)^{\alpha}(T o t-T b)^{\alpha}-(T-T b)^{2 \alpha}\right]}{(T o t-T b)^{2 \alpha}}
$$

quando $\mathrm{Tb} \leq \mathrm{T} \leq \mathrm{TB}$

$$
f(T)=0
$$

quando $\mathrm{T}<\mathrm{Tb}$ ou $\mathrm{T}>\mathrm{TB}$

$$
\alpha=\frac{\ln 2 / \ln (\mathrm{TB}-\mathrm{Tb})}{\mathrm{Tot}-\mathrm{Tb}}
$$

em que: Tb, Tot e TB são as temperaturas cardinais para aparecimento de nós em batata-doce $\mathrm{e} \mathrm{T}$ é a temperatura diária do $\operatorname{ar}\left({ }^{\circ} \mathrm{C}\right)$.

Diferentes combinações de temperaturas cardinais foram usadas no cálculo da STd nas Eqs. 3 e 4 e na f(T) nas Eqs. 7 
e 8 para determinar as temperaturas cardinais. Assim, foram testados $\mathrm{Tb}$ de $10,11,12,13,14$ e $15^{\circ} \mathrm{C}$, Tot de $28,29,30,31$ e $32{ }^{\circ} \mathrm{C}$ e TB de 40, 41, 42, 43 e $44{ }^{\circ} \mathrm{C}$ (Silva \& Lopes, 1995; Villavicencio et al., 2007; Noh et al., 2009; Ravi et al., 2009; Villordon et al., 2009).

Foram consideradas, ainda, duas versões do modelo PLASTO e de WE quanto à maneira como a temperatura do ar foi usada no cálculo da STd nas Eqs. 3 e 4 e na f(T) nas Eqs. 7 e 8. Na versão Tmed o cálculo da STd e de $\mathrm{f}(\mathrm{T})$ foi feito com a temperatura média diária do ar (TM) calculada pela média aritmética entre a TN e TX. Na versão Tmm foram calculados, separadamente a STd e a f(T) com a TN e com a TX calculandose, depois, a média aritmética das STd e das $\mathrm{f}(\mathrm{T}) \mathrm{s}$ diárias (Xue et al., 2004; Streck et al., 2009).

Os coeficientes PLAST e TANmax são dependentes do genótipo e foram estimados com dados de $\mathrm{NN}$ da cultivar Princesa, obtidos na época 2. Utilizou-se apenas uma época de plantio para calibrar os coeficientes a fim de que se tivesse o máximo de épocas com dados independentes e só então avaliar o desempenho dos modelos; foram utilizados dados independentes de NN coletados nas épocas 1, 3, 4, 5, 6 e 7. Na validação de modelos é importante que se tenha um número elevado de dados independentes, pois assim se tem mais segurança de que o modelo funciona. $\mathrm{O}$ desempenho dos modelos foi avaliado pelas seguintes estatísticas:

Raiz do quadrado médio do erro (RQME) (Janssen \& Heuberger, 1995):

$$
\mathrm{RQME}=\left[\frac{\sum(\mathrm{Si}-\mathrm{Oi})^{2}}{\mathrm{n}}\right]^{0,5}
$$

em que:

$\mathrm{Si}$ - valores simulados

Oi - valores observados

n - número de observações

Quanto menor é o valor dessa estatística, melhor será o modelo.

O QME (RQME $\left.{ }^{2}\right)$ foi decomposto em erro sistemático (QMEs) e não sistemático (QMEns) de acordo com Willmott (1981):

$$
\begin{aligned}
& \text { QMEs }=\frac{\left[\sum(\mathrm{Si}-\mathrm{Oi})^{2}\right]}{\mathrm{n}} \\
& \text { QMEns }=\frac{\left[\sum(\mathrm{Si}-\mathrm{Si})^{2}\right]}{\mathrm{n}}
\end{aligned}
$$

Baixo QMEs e alto QMEns são características de um bom modelo.

O índice BIAS expressa a tendência do modelo e varia de valores positivos quando há superestimativa e a negativos quando há subestimativa do modelo. Quanto mais próximo de zero, melhor o modelo (Leite \& Andrade, 2002):

$$
\mathrm{BIAS}=\frac{\sum \mathrm{Si}-\sum \mathrm{Oi}}{\sum \mathrm{Oi}}
$$

O coeficiente de correlação $(r)$ permite quantificar o grau de associação entre duas ou mais variáveis. $O$ valor de $r$ varia de -1 a 1 e quanto maior o valor absoluto de $r$ maior a adesão entre os valores observados e os simulados (Camargo \& Sentelhas, 1997):

$$
\mathrm{r}=\frac{\left[\sum(\mathrm{Oi}-\overline{\mathrm{O}})(\mathrm{Si}-\overline{\mathrm{S}})\right]}{\left\{\left[\sum(\mathrm{Oi}-\overline{\mathrm{O}})^{2}\right]\left[\sum\left(\mathrm{Si}-\overline{\mathrm{S}}^{2}\right)^{0,5}\right]\right\}}
$$

em que:

O - média dos valores observados e $\mathrm{S}$ é a média dos valores simulados

O índice de concordância $(\mathrm{dw})$ fornece a exatidão do modelo e seus valores variam de zero, para nenhuma concordância e a um para a concordância perfeita (Willmott, 1981):

$$
\mathrm{dw}=\frac{1-\left[\sum(\mathrm{Si}-\mathrm{Oi})^{2}\right]}{\left[\sum(|\mathrm{Si}-\overline{\mathrm{O}}|)+(|\mathrm{Oi}-\overline{\mathrm{O}}|)\right]^{2}}
$$

Fez-se a regressão entre valores observados e simulados a fim de observar a dispersão dos dados e se adotou o método proposto por Michel (1997), no qual os desvios (simulados menos observados) foram plotados com os valores observados, além de contabilizados os pontos que estavam fora de um intervalo aceitável de erro (envelope) de \pm 2 nós.

\section{Resultados E Discussão}

Nas diferentes épocas de plantio as plantas foram expostas a variadas condições de temperatura e radiação solar (Tabela 1). Nos experimentos envolvendo os anos 2010/2011, o mês mais frio foi junho de $2010\left(\mathrm{TN} 10,3^{\circ} \mathrm{C}\right.$ ) e o mês mais quente foi janeiro de 2011 ( $\mathrm{TX} 32,4^{\circ} \mathrm{C}$ ) enquanto nos anos 2011/2012 o mês mais frio foi agosto de 2011 (TN $10,9^{\circ} \mathrm{C}$ ) e o mês mais quente foi fevereiro de 2012 ( $\mathrm{TX} 33,1^{\circ} \mathrm{C}$ ). Durante todos os experimentos o mês de dezembro apresentou as maiores médias de radiação solar e os meses de menor radiação foram maio de 2010 e agosto de 2011. Essas diferentes condições meteorológicas afetaram a velocidade de emissão de nós e são relevantes para a calibração e avaliação de modelos matemáticos (Streck et al., 2007).

As estimativas dos coeficientes dos modelos com diferentes combinações de temperaturas cardinais sugerem que as temperaturas de 12 e $13{ }^{\circ} \mathrm{C}$ em combinação com 30 e $40{ }^{\circ} \mathrm{C}$ proporcionaram o melhor desempenho dos modelos, indicado pelo menor valor de RQME (Tabela 2). Esses resultados concordam com Noh et al. (2009), em que o desenvolvimento de plantas de batata-doce expostas a temperaturas de $12{ }^{\circ} \mathrm{C}$ foi severamente prejudicado com redução acentuada no número de folhas e crescimento da planta. Temperaturas de 14 e $15^{\circ} \mathrm{C}$ são citadas como limitantes ao crescimento das raízes tuberosas, que têm exigências térmicas diferentes das do desenvolvimento vegetativo (Ravi et al., 2009; Villordon et al., 2009). Temperatura de $30{ }^{\circ} \mathrm{C}$ favorece o desenvolvimento da parte 
Tabela 1. Média mensal da temperatura mínima (TN) e máxima (TX) do ar e radiação solar global incidente durante o período experimental nos anos 2010, 2011 e 2012 em Santa Maria, RS, Brasil

\begin{tabular}{llccc}
\hline \multirow{2}{*}{ Ano } & \multicolumn{1}{c}{ Mês } & \multicolumn{2}{c}{ Temperatura } & \multicolumn{2}{c}{$\begin{array}{c}\text { Radiação } \\
\text { solar global } \\
\text { ( } \mathbf{C} \text { C) }\end{array}$} & $\begin{array}{c}\text { TN } \\
\text { (MJ m }^{-2} \mathbf{d ~}^{-1} \text { ) }\end{array}$ \\
\cline { 3 - 4 } 2010 & Março & 19,2 & 30,1 & 15,7 \\
2010 & Abril & 14,2 & 26,1 & 12,3 \\
2010 & Maio & 12,6 & 21,0 & 7,9 \\
2010 & Junho & 10,8 & 19,9 & 7,9 \\
2010 & Outubro & 12,7 & 24,4 & 16,7 \\
2010 & Novembro & 14,9 & 27,7 & 19,1 \\
2010 & Dezembro & 18,4 & 30,4 & 21,2 \\
2011 & Janeiro & 21,8 & 32,5 & 19,5 \\
2011 & Fevereiro & 20,9 & 30,0 & 17,3 \\
2011 & Março & 18,2 & 28,8 & 16,3 \\
2011 & Agosto & 10,9 & 19,9 & 10,2 \\
2011 & Setembro & 11,1 & 23,5 & 13,9 \\
2011 & Outubro & 14,6 & 25,2 & 16,2 \\
2011 & Novembro & 16,1 & 29,0 & 19,4 \\
2011 & Dezembro & 17,2 & 30,3 & 20,3 \\
2012 & Janeiro & 19,5 & 33,0 & 18,0 \\
2012 & Fevereiro & 21,2 & 33,1 & 19,1 \\
2012 & Março & 17,2 & 30,3 & 15,5 \\
2012 & Abril & 13,7 & 25,3 & 13,4 \\
2012 & Maio & 11,9 & 24,8 & 11,4 \\
\hline
\end{tabular}

aérea da planta enquanto temperatura de $25^{\circ} \mathrm{C}$ é considerada ótima para o crescimento da raiz (Villavicencio et al., 2007). Já em temperaturas supra ótimas o desenvolvimento vegetativo se mantém até $40^{\circ} \mathrm{C}$, o que não ocorre com o crescimento das raízes (Ravi et al., 2009). Desta forma, as temperaturas de 12, 30 e $40{ }^{\circ} \mathrm{C}$ foram consideradas as temperaturas cardinais $(\mathrm{Tb}$, Tot e TB, respectivamente) nos dois modelos (PLASTO e WE). Mencionadas temperaturas foram usadas para o cálculo dos coeficientes PLAST e TANmax, estimados com os dados da época 2 , em 23,8 e $19,8^{\circ} \mathrm{C} d$ nó ${ }^{-1}$ e 0,698 e 0,824 nós $\mathrm{d}^{-1}$, nas versões Tmed e Tmm, respectivamente.

A RQME foi, para o modelo PLASTO, 2,1 e 4,1 nós haste ${ }^{-1}$ na versão Tmed e Tmm e 2,2 e 3,5 nós haste ${ }^{-1}$ para o modelo de WE na versão Tmed e Tmm, respectivamente (Tabela 2). Esses valores sugerem melhor desempenho do modelo PLASTO versão Tmed, embora a diferença para o modelo de WE nesta versão tenha sido pequena $\left(0,1\right.$ nós haste $\left.{ }^{-1}\right)$. Os modelos usados neste estudo simularam melhor o número de nós que os modelos MADHURAM (Somasanduran \& Mithra, 2008) e SPOTCOMS (Mithra \& Somasanduran, 2008), em que os erros variaram de 5,8 até 19,3 nós.

O componente sistemático (QMEs) para o modelo de WE nas versões Tmed e Tmm foi menor (3,3 e 13,7\%, respectivamente) que no modelo PLASTO (11,1 e 19,5\%,

Tabela 2. Valores dos coeficientes PLAST $\left({ }^{\circ} \mathrm{C} d\right.$ nó $\left.^{-1}\right)$ e TANmáx (nós $\mathrm{d}^{-1}$ ) e a raiz do quadrado médio do erro (RQME) da simulação do número de nós na haste principal da planta de batata-doce, cultivar Princesa, nas épocas de plantio usadas como dados independentes com diferentes combinações de temperaturas cardinais, em duas versões (Tmed e Tmm) dos modelos do plastocrono (PLASTO) e de Wang e Engel (WE)

\begin{tabular}{|c|c|c|c|c|c|c|c|c|c|c|}
\hline \multirow{2}{*}{\multicolumn{3}{|c|}{$\begin{array}{c}\text { Temperaturas cardinais } \\
\left({ }^{\circ} \mathrm{C}\right)\end{array}$}} & \multicolumn{4}{|c|}{ PLASTO } & \multicolumn{4}{|c|}{ WE } \\
\hline & & & & & & & & & & \\
\hline Tb & Tot & TB & PLAST & RQME & PLAST & RQME & TANmáx & RQME & TANmáx & RQME \\
\hline 10 & 28 & 40 & 26,80 & 2,8 & 20,20 & 5,4 & 0,621 & 4,0 & 0,733 & 4,7 \\
\hline 10 & 29 & 40 & 27,30 & 2,7 & 21,60 & 4,9 & 0,648 & 3,1 & 0,762 & 4,3 \\
\hline 10 & 30 & 40 & 27,30 & 2,6 & 22,90 & 4,6 & 0,689 & 2,6 & 0,802 & 3,8 \\
\hline 10 & 31 & 40 & 27,30 & 2,6 & 24,20 & 4,2 & 0,753 & 2,5 & 0,857 & 3,3 \\
\hline 10 & 32 & 40 & 27,30 & 2,6 & 25,10 & 3,9 & 0,853 & 2,5 & 0,934 & 2,8 \\
\hline 11 & 28 & 40 & 25,10 & 2,6 & 18,70 & 5,2 & 0,623 & 3,7 & 0,742 & 4,6 \\
\hline 11 & 29 & 40 & 25,50 & 2,4 & 20,00 & 4,7 & 0,651 & 2,9 & 0,771 & 4,2 \\
\hline 11 & 30 & 40 & 25,60 & 2,3 & 21,40 & 4,3 & 0,693 & 2,4 & 0,812 & 3,7 \\
\hline 11 & 31 & 40 & 25,60 & 2,3 & 22,50 & 4,0 & 0,759 & 2,3 & 0,868 & 3,3 \\
\hline 11 & 32 & 40 & 25,50 & 2,3 & 23,50 & 3,7 & 0,862 & 2,7 & 0,946 & 2,8 \\
\hline 12 & 28 & 40 & 23,40 & 2,3 & 17,20 & 4,9 & 0,625 & 3,4 & 0,752 & 4,4 \\
\hline 12 & 29 & 40 & 23,80 & 2,1 & 18,50 & 4,5 & 0,654 & 2,7 & 0,796 & 4,0 \\
\hline 12 & 30 & 40 & 23,80 & 2,1 & 19,80 & 4,2 & 0,698 & 2,2 & 0,824 & 3,5 \\
\hline 12 & 31 & 40 & 23,80 & 2,1 & 20,90 & 3,9 & 0,766 & 2,3 & 0,881 & 3,1 \\
\hline 12 & 32 & 40 & 23,80 & 2,1 & 21,80 & 3,6 & 0,872 & 2,7 & 0,960 & 2,9 \\
\hline 13 & 28 & 40 & 21,70 & 2,2 & 15,70 & 4,6 & 0,630 & 3,1 & 0,763 & 4,2 \\
\hline 13 & 29 & 40 & 22,10 & 2,1 & 16,90 & 4,3 & 0,658 & 2,4 & 0,795 & 3,8 \\
\hline 13 & 30 & 40 & 22,10 & 2,1 & 18,20 & 4,0 & 0,700 & 2,2 & 0,837 & 3,4 \\
\hline 13 & 31 & 40 & 22,10 & 2,1 & 19,30 & 3,7 & 0,775 & 2,5 & 0,896 & 3,0 \\
\hline 13 & 32 & 40 & 22,10 & 2,1 & 20,20 & 3,4 & 0,880 & 3,2 & 0,976 & 2,7 \\
\hline 14 & 28 & 40 & 20,00 & 2,4 & 14,20 & 4,4 & 0,632 & 2,7 & 0,777 & 4,0 \\
\hline 14 & 29 & 40 & 20,30 & 2,3 & 15,50 & 4,1 & 0,663 & 2,3 & 0,809 & 3,7 \\
\hline 14 & 30 & 40 & 20,40 & 2,3 & 16,60 & 3,9 & 0,711 & 2,3 & 0,853 & 3,4 \\
\hline 14 & 31 & 40 & 20,40 & 2,3 & 17,70 & 3,7 & 0,784 & 2,8 & 0,913 & 2,9 \\
\hline 14 & 32 & 40 & 20,40 & 2,3 & 18,50 & 3,4 & 0,909 & 3,6 & 0,994 & 2,7 \\
\hline 15 & 28 & 40 & 18,30 & 2,8 & 12,80 & 4,3 & 0,636 & 2,5 & 0,795 & 3,9 \\
\hline 15 & 29 & 40 & 18,60 & 2,8 & 13,90 & 4,0 & 0,669 & 2,3 & 0,828 & 3,6 \\
\hline 15 & 30 & 40 & 18.70 & 2,8 & 15,10 & 3.8 & 0,719 & 2.6 & 0.873 & 3.3 \\
\hline 15 & 31 & 40 & 18,70 & 2,8 & 16,10 & 3,6 & 0,796 & 3,2 & 0,934 & 2,9 \\
\hline 15 & 32 & 40 & 18,70 & 2,8 & 16,90 & 3,3 & 0,916 & 4,0 & 1,016 & 2,6 \\
\hline
\end{tabular}


respectivamente) indicando que o modelo de WE é superior nesta estatística. Os valores das estatísticas dw e $\mathrm{r}$ foram elevados (acima de 0,95 ) em todos os modelos e versões sendo maior nos modelos da versão Tmed. Considerando esta versão, conclui-se que não houve diferenças acentuadas entre PLASTO e WE para os valores de dw $(0,993$ e 0,992$)$ e r $(0,986$ e 0,985). Para os valores de BIAS ocorreu uma subestimação do NN para a versão Tmm cujos modelos na versão Tmed apresentaram os valores mais próximos a zero (Tabela 3 ).

A análise visual dos dados observados e simulados sugere melhor desempenho para os modelos na versão Tmed sendo que tanto para PLASTO como para WE, os valores simulados estão próximos da linha 1:1 (Figuras 2A e 2B). O valor do resíduo, apresentado nas inserções de cada painel da Figura 2 também foi semelhante entre os dois modelos nesta versão. Os desvios variaram de 4,7 a -6,8 nós haste ${ }^{-1}$ para o PLASTO e 5,9 a -6,5 nós haste ${ }^{-1}$ para WE. Valores mais elevados são apresentados na
Tabela 3. Valores das estatísticas raiz do quadrado médio do erro (RQME), erro sistemático (QMEs), erro não sistemático (QMEns), índice BIAS, índice de concordância (dw) e coeficiente de correlação (r) utilizados na avaliação de duas versões (Tmed e Tmm) dos modelos do plastocrono (PLASTO) e de Wang e Wengel (WE) na simulação da emissão de nós na haste principal da planta de batata-doce, cultivar Princesa, considerando-se as temperaturas cardinais de 12,30 e $40{ }^{\circ} \mathrm{C}$

\begin{tabular}{lcccc}
\hline \multirow{2}{*}{ Estatística } & \multicolumn{2}{c}{ Tmed } & \multicolumn{2}{c}{ Tmm } \\
\cline { 2 - 5 } RQME (nós) & PLASTO & WE & PLASTO & WE \\
QMEs (\%) & 2,1 & 2,20 & 4,10 & 3,50 \\
QMEns (\%) & 11,1 & 3,30 & 19,50 & 13,70 \\
BIAS & 88,9 & 96,70 & 80,50 & 86,30 \\
DW & 0,003 & 0,002 & $-0,011$ & $-0,007$ \\
R & 0,993 & 0,992 & 0,972 & 0,979 \\
\hline
\end{tabular}
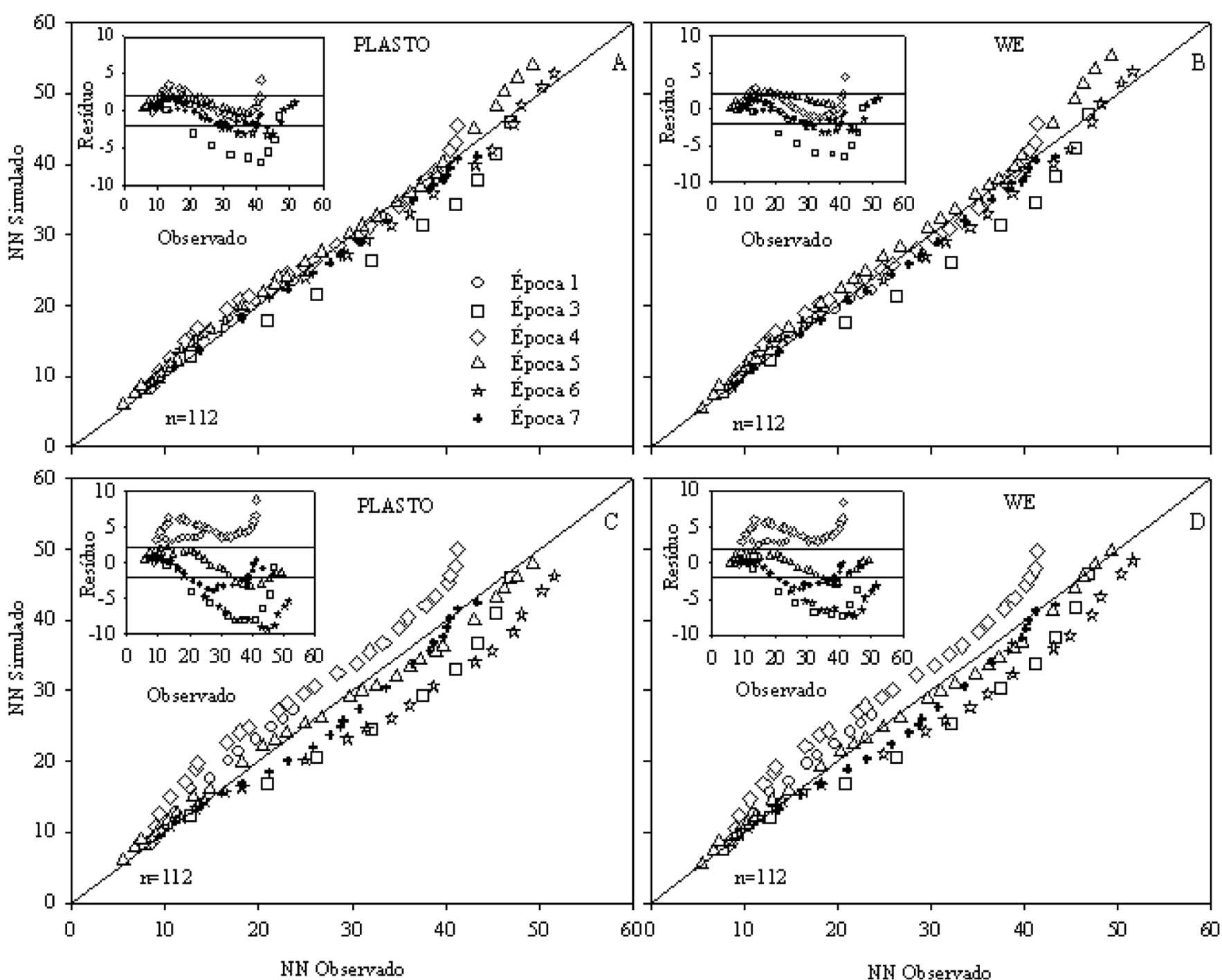

Obs.: 0 resíduo (NN simulados menos NN observados) versus o NN observados é apresentado nas inserções, com envelope de \pm 2 nós

Figura 2. Número de nós (NN) observados versus simulados na haste principal da planta de batata-doce, cultivar Princesa, nas épocas de plantio usadas como dados independentes (27/03/2010, 21/12/2010, 25/08/2011, 21/11/2011, 03/01/2012 e 13/02/2012, representando as épocas 1, 3, 4, 5, 6 e 7, respectivamente) pelos modelos do plastocrono (PLASTO) e de Wang e Wengel (WE) em duas versões de temperatura do ar (Tmed: A e B; Tmm: C e D), considerando as temperaturas cardinais de 12,30 e $40^{\circ} \mathrm{C}$. A linha cheia é a linha $1: 1$ 
versão Tmm variando de 8,8 a -9 nós haste-1 ${ }^{-1}$ ara o PLASTO e 8,4 a $-7,3$ nós haste ${ }^{-1}$ para WE.

Assumindo um erro aceitável de dois nós, semelhante ao que foi assumido por Streck et al. (2006) para meloeiro, no total de 112 pontos, observa-se que o número de pontos com desvios iguais ou maiores que dois, foi de 30 (27\%) para o PLASTO e 32 (28\%) para WE na versão Tmed confirmando, mais uma vez, a performance similar de ambos. Na versão Tmm o número de pontos fora do intervalo aceitável foi 71 (63\%) para o PLASTO e de $67(60 \%)$ para WE.

$\mathrm{Na}$ análise para cada época de plantio o RQME foi maior para os modelos na versão Tmm (Tabela 4), com exceção da época 5 (plantio em 21/11/2011), em que os modelos tiveram melhor desempenho destacando-se o modelo de WE. Para as outras épocas o RQME variou de 2,22 a 5,81 nós haste ${ }^{-1}$. Na versão Tmed os valores variaram de 1,21 a 2,41 nós haste ${ }^{-1}$, com exceção da época 3 em que os valores foram os mais elevados. Acredita-se que o maior erro encontrado nesta época seja devido ao aumento da velocidade de emissão de nós, em virtude das irrigações mais frequentes realizadas. De acordo com Sassaki \& Machado (1999) maior disponibilidade de água no solo leva a um aumento da condutância estomática e da transpiração. Com isto, maior é o suprimento de $\mathrm{CO}_{2}$ intracelular necessário para a

Tabela 4. Raiz do quadrado médio do erro (RQME) da simulação da emissão de nós na haste principal da planta de batata-doce, cultivar Princesa, com os modelos plastocrono (PLASTO) e Wang e Wengel (WE) em duas versões (Tmed e Tmm) em cada época de plantio usada como dado independente

\begin{tabular}{ccccc}
\hline \multirow{2}{*}{ Época } & \multicolumn{4}{c}{ RQME (nós haste- ${ }^{-1}$ ) } \\
\cline { 2 - 5 } & \multicolumn{3}{c}{ Tmed } & \multicolumn{3}{c}{ Tmm } \\
\cline { 2 - 5 } $1(27 / 03 / 2010)$ & PLAST0 & WE & PLASTO & WE \\
$3(21 / 12 / 2010)$ & 1,31 & 1,21 & 3,05 & 2,52 \\
$4(25 / 08 / 2011)$ & 4,34 & 4,28 & 5,47 & 4,92 \\
$5(21 / 11 / 2011)$ & 1,87 & 1,69 & 5,01 & 4,63 \\
$6(03 / 01 / 2012)$ & 1,83 & 2,41 & 1,90 & 1,42 \\
$7(13 / 02 / 2012)$ & 1,94 & 1,90 & 5,81 & 4,45 \\
& 1,31 & 1,35 & 2,37 & 2,22 \\
\hline
\end{tabular}

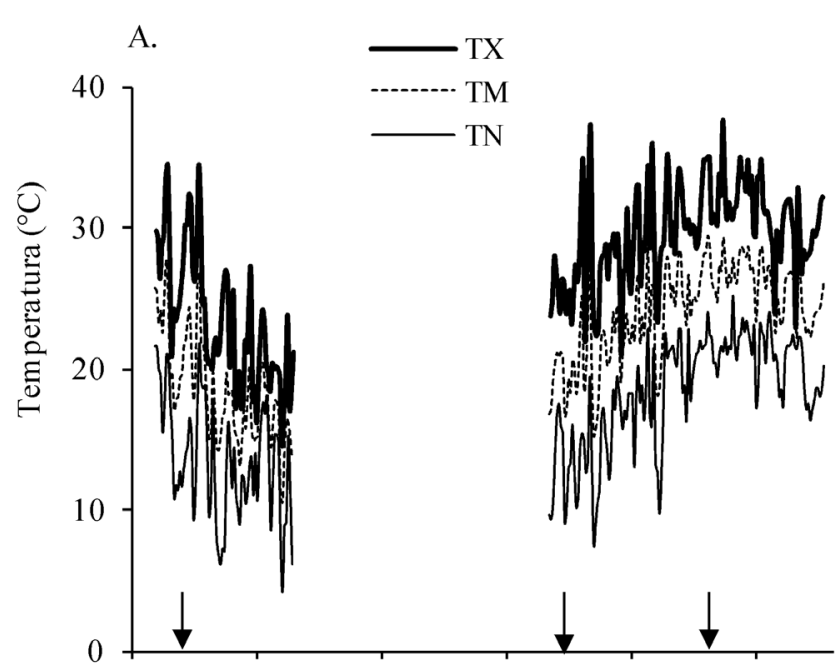

15/3/10 19/5/10 23/7/10 26/9/10 30/11/10 3/2/1

Dias ocorrência da fotossíntese e produção de carboidratos com vista ao desenvolvimento da planta. Considerando a versão Tmed, não há diferenças acentuadas entre WE e PLASTO nas diversas épocas sendo que as maiores diferenças de RQME foram de 0,$1 ; 0,18$ e 0,58 nós haste ${ }^{-1}$ nas épocas 1,4 e 5 , respectivamente.

Os resultados encontrados sugerem que o uso da TN e TX nos modelos PLASTO e WE não melhorou a simulação do NN comparado com o uso da TM. Esses resultados contrastam com os de Streck et al. (2009) que encontraram melhora na predição da velocidade de aparecimento de folhas em milho com a versão Tmm no modelo de WE mas concordam com Xue et al. (2004), em que o uso da TM promoveu melhor previsão de Estágio Haun em trigo de inverno que com o uso da TN e TX no modelo de WE. Sugere-se, então, o uso da TM como dado de entrada dos modelos PLASTO e WE na cultura da batatadoce, por ser uma abordagem mais simples.

$\mathrm{Na}$ versão Tmed os dois modelos tiveram desempenho semelhante. Uma provável explicação para esses resultados é que tanto PLASTO quanto WE tendem a se assemelhar na resposta da emissão de nós em uma grande faixa de temperatura do ar entre a Tb e a Tot (Figura 1). Quando o ciclo da cultura ocorre na época recomendada geralmente as temperaturas se enquadram nesta faixa, caso em que os dois modelos funcionam bem (Streck et al., 2005).

A batata-doce é uma planta perene de origem tropical e, em condições subtropicais, o cultivo é sazonal. Segundo Castro (2010), no Rio Grande do Sul seu plantio pode ser realizado durante a primavera e o verão, entre os meses de outubro a janeiro e o ciclo pode estender-se até o outono. As épocas 2, $3,5,6$ e 7 utilizadas neste trabalho se enquadram no período recomendado no qual as temperaturas médias diárias do ar estiveram quase sempre abaixo da temperatura ótima $\left(30^{\circ} \mathrm{C}\right)$, com exceção de um dia, em 11 de janeiro de 2012, e dois dias, em 16 e 17 de fevereiro de 2012 (Figura 3B). As épocas 1 e 4, realizadas fora do período recomendado, tiveram temperaturas baixas e próximas à $\mathrm{Tb}$ no início da época 1 (Figura $3 \mathrm{~A}$ ) e no

B.

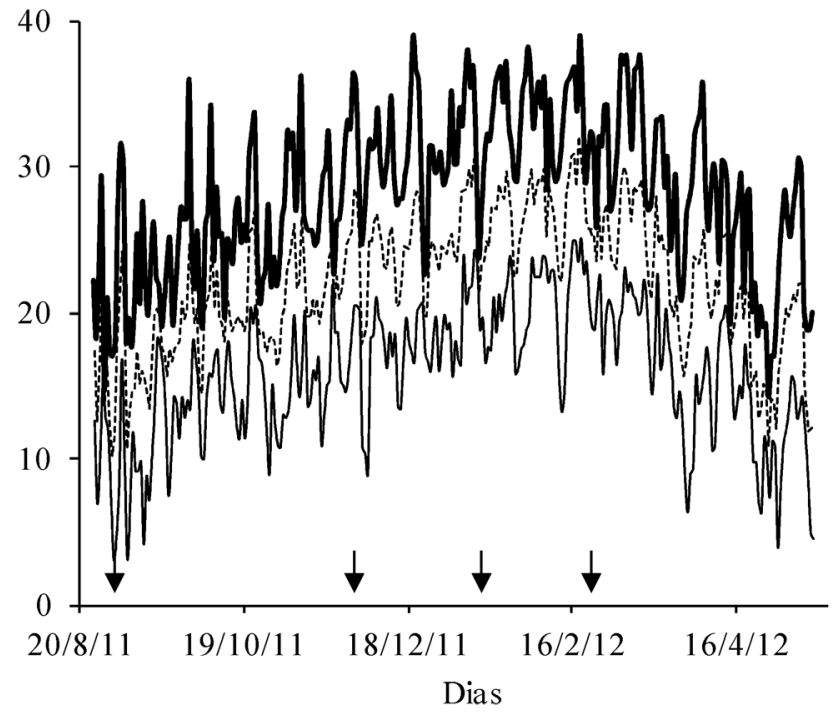

Figura 3. Temperaturas máxima (TX), média (TM) e mínima (TN) diária do ar durante o período experimental nos anos de 2010/2011 (A) e 2011/2012 (B). As setas indicam as datas de plantio (Épocas 1 a 7) 
final da época 4 (Figura 3B); neste caso, o modelo de WE foi ligeiramente superior (Tabela 4).

Modelos que utilizam funções não lineares de resposta à temperatura, são reconhecidos como superiores à soma térmica para descrever o desenvolvimento de várias espécies (Streck, 2003; Xue et al., 2004; Streck et al., 2007; 2008a). Mas resultados relatados por Streck et al. (2011) mostram desempenho similar entre o modelo de WE e da soma térmica para simular o desenvolvimento em cultivares de arroz. Os autores destacam que esta ocorrência se deve ao fato das épocas de cultivo apresentarem temperaturas na faixa linear de desenvolvimento do arroz. Em outro trabalho com a cultura do melão o valor de RQME foi similar para os modelos PLASTO e WE porém o modelo de WE foi melhor pelo menor valor dos resíduos (Streck et al., 2006). No presente trabalho até mesmo os resíduos foram semelhantes.

Vários fatores contribuem para a escolha de um modelo, dentre os quais se destacam a simplicidade, a facilidade de obtenção dos dados de entrada e, principalmente, o significado biológico e a definição operacional dos coeficientes (Streck et al., 2011). Tanto PLASTO como WE são considerados modelos de fácil implementação; no entanto, por usar uma $\mathrm{f}(\mathrm{T})$ nãolinear, o modelo de WE é mais recomendado para descrever o desenvolvimento em resposta à temperatura haja vista que funciona adequadamente em diferentes condições térmicas. Além disto, apresenta a vantagem de combinar os efeitos do ambiente sobre a TAN de forma multiplicativa (Streck et al., 2003). Assim, o modelo de WE pode ser uma alternativa ao modelo PLASTO em situações em que este não funcione adequadamente. Em cenários climáticos futuros, em que a temperatura do ar é esperada acima da atual, espera-se que a planta de batata-doce seja exposta a temperaturas acima da Tot com mais frequência (IPCC, 2007) e neste caso o modelo de WE pode descrever melhor o desenvolvimento.

\section{Conclusões}

1. Melhor predição do número de nós em batata-doce é obtida com o uso das temperaturas cardinais de 12,30 e $40^{\circ} \mathrm{C}$.

2. Os modelos plastocrono e Wang e Engel apresentam desempenho semelhante e podem ser utilizados para simular o desenvolvimento vegetativo da batata-doce quando cultivada na época recomendada. Fora deste período sugere-se usar o modelo de Wang e Engel.

3. A versão dos modelos plastocrono e Wang e Engel para batata-doce que usa a temperatura média é superior à versão desses modelos que usam a temperatura mínima e máxima diária do ar como dados de entrada.

\section{Literatura Citada}

Arnold, C. Y. Maximum-minimum temperatures as a basis for computing heat units. Proceedings of the American Society for Horticultural Sciences, v.76, p.682-692, 1960.

Baker, J. T.; Reddy, V. R. Temperature effects on phenological development and yield of muskmelon. Annals of Botany, v.87, p.605-613, 2001.
Camargo, A. P.; Sentelhas, P. C. Avaliação do desempenho de diferentes métodos de estimativa da evapotranspiração potencial no Estado de São Paulo, Brasil. Revista Brasileira de Agrometeorologia, v.5, p.89-97, 1997.

Castro, L. A. S. Instruções para plantio de mudas de batata-doce com alta sanidade. Pelotas: Embrapa Clima Temperado, 2010. 19p. Documentos, 313

IPCC - Intergovernmental Panel on Climate Change. Climate change 2007: The physical science basis. Contribution of Working Group I to the Fourth Assessment Report of the Intergovernmental Panel on Climate Change [Solomon, S.; D. Qin, M.; Manning, Z.; Chen, M.; Marquis, K. B.; Averyt, M.;Tignor; H. L. Miller (ed.)]. Cambridge: Cambridge University Press, New York:United Kingdom,2007. 996p.

Janssen, P. H. M.; Heuberger, P. S. C. Calibration of processoriented models. Ecological Modelling, v.83, p.55-56, 1995.

Leite, H. G.; Andrade, V. C. L. Um método para condução de inventários florestais sem o uso de equações volumétricas. Revista Árvore, v.26, p.321-328, 2002.

Michel, P. L. Misuse of regression for empirical validation of models. Agricultural Systems, v.54, p.313-326, 1997.

Mithra, V. S.; Somasundaram, K. A model to simulate Sweet Potato Growth. World Applied Sciences Journal, v.4, p.568577, 2008.

Noh, S. A.; Huh, G. H.; Shin, J. S. Growth retardation and differential regulation of expansin genes in chilling-stressed sweetpotato. Plant Biotechnol Reports, v.3, p.75-85, 2009.

Ravi, V.; Naskar, S. K.; Makeshkumar, T.; Binoy, B.; Krishnan, P. B. S. Molecular physiology of storage root formation and development in sweet potato (Ipomoea batatas (L.) Lam.). Journalof Root Crops, v.35, p.1-27, 2009.

Sassaki, R. M.; Machado, E. C. Trocas gasosas e condutância estomática em duas espécies de trigo em diferentes teores de água no solo. Pesquisa Agropecuária Brasileira, v.34, p.1571-1579, 1999.

Silva, J. B. C ; Lopes, C. A. Cultivo de batata- doce [Ipomoea batatas (L.) Lam.]. Brasília: Embrapa CNPH, 1995. 18p. Instruções Técnicas - CNPH, 7.

Somasundaram, K.; Mithra, V.S. MADHURAM: A simulation model for sweet potato growth. World Journal of Agricultural Sciences, v.4, p.241-254, 2008.

Streck, E. V.; Kampf, N.; Dalmolin, R. S. D.; Klamt, E.; Nascimento, P. C.; Schneider, P.; Giasson, E.; Pinto, L. F. S. Solos do Rio Grande do Sul. Porto Alegre: EMATER/ RS; UFRGS, 2008a. 126p.

Streck, N. A. A generalized nonlinear temperature response function for some growth and developmental parameters in kiwifruit (Actinidia deliciosa (A. Chev.) C. F. Liang \& A. R. Ferguson). Ciência Rural, v.33, p.255-262, 2003.

Streck, N. A.; Gabriel, L. F.; Samboranha, F. K.; Lago, I.; Schwantes, A. P.; Schons, A. Comparing two versions of a non-linear model for simulating leaf number and developmental stages in maize based on air temperature. Ciência Rural, v.39, p.642-648, 2009. 
Streck, N. A.; Lago, I.; Buriol, G. A.; Heldwein, A. B.; Tibola, T.A nonlinear model to simulate node appearance in muskmelon (Cucumis melo L.) grown inside plastic greenhouse as a function of air temperature. RevistaBrasileira de Agrometeorologia, v.14, p.210-216, 2006.

Streck, N. A.; Lago, I.; Gabriel, L. F.; Samboranha, F. K. Simulating maize phenology as a function of air temperature with a linear and a non linear model. Pesquisa Agropecuária Brasileira, v.43, p.449-455, 2008.

Streck, N. A.; Lago, I.; Oliveira, F. B.; Heldwein, A. B.; Avila, de L. A.; Bosco, L. C. Modeling the development of cultivated rice and weedy red rice. American Society of Agricultural and Biological Engineers, v.54, p.371-384, 2011.

Streck, N. A.; Paula, F. L. M.; Bisognin, D. A.; Heldwein, A. B.; Dellai, J. Simulating the development of field grown potato (Solanum tuberosum L.) Agricultural and Forest Meteorology, v.142, p.1-11, 2007.

Streck, N. A.; Tibola, T.; Lago, I. ; Buriol, G. A.; Heldwein, A. B.; Schneider, F. M.; Zago, V. Estimativa do plastocrono em meloeiro (Cucumis melo L.) cultivado em estufa plástica em diferentes épocas do ano. Ciência Rural, v.35, p.12751280, 2005.

Streck, N. A.; Weiss, A.; Xue, Q.; Baenziger, S. P. Improving predictions of developmental stages in winter wheat: a modified Wang and Engel model. Agricultural and Forest Meteorology, v.115, p.139-150, 2003.
Villavicencio, L. E. ; Blankenship, S. M.; Yencho, G. C.; Thomas, J. F.; Raper, C. D. Temperature effect on skin adhesion, cell wall enzyme activity, lignin content, anthocyanins, growth parameters, and periderm histochemistry of sweet potato. Journal of the American Society for Horticultural Science, v.132, p.729-738, 2007.

Villordon, A.; Clark, C.; Ferrin, D.; LaBonte, D. Using growing degree days, climatic variables, linear regression, and data mining methods to help improve prediction of sweetpotato harvest date and yield in Louisiana. HortTechnology, v.19, p.133-144, 2009.

Walter, L. C.; Rosa, H. T.; Streck, N. A. Simulação do rendimento de grãos de arroz irrigado em cenários de mudanças climáticas. Pesquisa Agropecuária Brasileira, v.45, p.1237-1245, 2010.

Wang, E.; Engel, T. Simulation of phenological development of wheat crops. Agricultural Systems, v.58, p.1-24, 1998.

Willmott, C. J. On the validation of models. Physical Geograph, v.2, p.184-194, 1981.

Xue, Q.; Weiss, A.; Baenziger, S. P. Predicting leaf appearance in field-grown winter wheat: Evaluating linear and non-linear models. Ecological Modeling, v.175, p.261-270, 2004.

Yan, W. K.; Hunt, L. A. An equation for modeling the temperature response of plants using only the cardinal temperatures. Annals of Botany, v.84, p.607614,1999 . 